\title{
Private Provision of Public Goods and Information Diffusion in Social Groups*
}

\author{
Kimberley Scharf \\ University of Warwick and CEPR
}

This version: November 2011

\begin{abstract}
We describe a model of costly information sharing in social groups, where private information about quality of provision is transmitted by social proximity. Individuals engage in voluntary provision of a pure collective good that is consumed by both neighbours and non-neighbours. We show that, unlike in the case of private goods, better informed individuals face positive incentives to incur a cost to share information with their neighbours. These incentives are stronger, and provision of the pure public good greater, the smaller are individuals' social neighbourhoods.
\end{abstract}

KEY WORDS: Private provision of public goods, social learning JEL CLASSIFICATION: H1, L3, D6, D7

*Comments and correspondence should be addressed to Kimberley Scharf, Department of Economics, University of Warwick, Coventry CV4 7AL, UK, k.scharf@warwick.ac.uk 


\section{Introduction}

There is evidence that social interactions matter for voluntary contributions towards collective goods (Brown and Ferris, 2007). Casual empiricism suggests the same - being targeted by co-workers and acquaintances for fundraising towards various charitable causes is a commonplace experience. Sociological research has long stressed the role of social links in motivating individual behaviour (e.g. Wasserman and Faust, 1994), but has paid little attention to the role of social connections in voluntary giving - a notable exception being Galaskiewicz (1985). Although much recent work in economics has focused on the role of interpersonal links in motivating economic behaviour in general, and voluntary giving in particular, to the best of our knowledge there has been no investigation of how social interactions affect giving towards third parties, and specifically of the question of whether more social interaction leads to more voluntary giving.

This paper tries to fill this gap, formalizing the relationship between voluntary social interactions, information sharing and contribution equilibria. It describes a social proximity-based mechanism of information transmission in groups of individuals who consume a pure public good. In the mechanism we study, information about quality for alternative modes of provision of a public good can spread from one individual to the next just as it does for private goods. However, unlike in the case of private goods, better informed individuals face positive incentives to incur private costs in order to transmit information to their less informed neighbours, because this can bring about an increase in collective provision, the benefits of which they partake in. In this setting, the sharing of information has the characteristics of a local public good that is confined within individual social neighbourhoods, even when voluntary contributions fund the provision of a pure public good that spans all neighbourhoods. Thus, incentives to engage in costly information sharing are stronger when social neighbourhoods are smaller; conse- 
quently, large societies composed of comparatively small social neighbourhoods can sustain comparatively higher levels of private provision of collective goods.

These conclusions are in line with the view that individuals are more "engaged" in collective choices in smaller communities than they are in larger communities; ${ }^{1}$ but our analysis delivers a new theoretical argument for why the same conclusions may extend to collective choices that are not local in nature.

Understanding how and why social connections can shape voluntary giving also has implications for understanding how government policies affect private giving. As many developed countries are increasing their reliance on the private sector to meet collective needs, we see a shift in the use of public resources from the funding of public provision to the subsidization of private provision. Our findings suggest that, in designing such subsidies, policymakers may be able to leverage on the relationship between private giving and social structure to maximize their impact; specifically, targeted subsidies towards fundraising effort at the local level may be an effective way of promoting private giving at the central level.

Our paper is related to two main strands of literature: the literature on private contributions towards collective consumption (Malinvaud, 1972; Bergstrom, Blume and Varian, 1986; Andreoni, 1990; and subsequent contributions); and the literature on social learning (Banerjee, 1992; Ellison and Fudenberg, 1995; and subsequent contributions). Two recent papers that are somewhat related to ours are Dutta and Chatterjee (2010), and Bramoullé and Kranton (2007). The first paper looks looks at costless information transmission across consumers for the case of private goods; as we have already noted, the public good case is fundamentally different from the private good case - where no costly transmission of informa-

\footnotetext{
${ }^{1}$ For example, a 2010 report on volunteering and charitable giving by the UK Department for Communities and Local Government concludes that rural dwellers are significantly more likely to engage in volunteering than urban dwellers are.
} 
tion can occur. The second paper focuses on the provision of public goods in networks, with the structure of network links determining the scope of local public goods, and fully abstracting from voluntary transmission of information. Our line of questioning is quite different: we specifically study voluntary information sharing, and focus on a scenario where the collective good provided is a pure public good - whose scope is independent of social links - and where social links are only relevant for information transmission.

The paper is organized as follows. Section 2 develops a dynamic model of private giving and costly information sharing in social groups. Section 3 derives results concerning the relationship between neighbourhood size, information, and information sharing. Section 4 discusses an alternative but equivalent specification, where private giving is uniquely motivated by private "warm-glow" effects; it also presents an extension where individuals have different preferences with respect to collective consumption. Section 6 derives implications for policy design. Section 7 summarizes and concludes.

\section{A model of private giving and information sharing in social neighbourhoods}

The theoretical literature on the voluntary provision of public goods has highlighted two motives that may lie behind private giving: consumption motives and outwardly orientated motives (e.g. "warm glow" or status signaling), with the latter typically having to be invoked whenever the former is unable to account for giving in large groups. In this paper we describe an information-transmission mechanism of contagion in private giving, which can be related to both private consumption motives and warm-glow motives, and is also related to the transmission mechanism that is stressed by the literature on social learning. 
We examine incentives on both sides of a given social link between two socially connected individuals, and then derive implications for the diffusion of philanthropic behaviour through a social group. Our analysis deliberately abstracts (by way of suitable assumptions) from the topological structure of social links. ${ }^{2}$

The dynamic model we develop is built on assumptions that lead to stationary outcomes, making it possible to characterize the "long-run" relationship between the density of social interactions, information transmission and collective provision outcomes. The model is highly stylized and abstracts from many other dimensions of heterogeneity that are relevant to real-world voluntary giving to concentrate on how the density of social interactions affects the sharing of provisionrelevant information that is not publicly observable.

\section{Preferences, technologies and information structure}

There is an economy with $N$ individuals, $i \in I \equiv\{1, \ldots, N\}$, and a countable number of periods indexed by $t=1,2, \ldots$. Each individual consumes both a private good and a pure public good in each period, in amounts that are respectively denoted by $x_{i, t}$ and $G_{t}$. Provision of the public good in period $t$ is funded by private contributions, $v_{i, t}$, made in each period by each individual $i \in I$, out of her exogenously given period- $t$ income, $y_{i, t}$.

The public good is produced at a marginal cost of unity by $M$ private non-profit providers (charities), $j \in J \equiv\{1, \ldots, M\}$, and sold at marginal cost. Individuals can make contributions to a single provider in each period. Providers differ from each other only with respect to the quality of their provision, $q_{j, t} \in\{\bar{q}, q\}$, with

\footnotetext{
${ }^{2}$ Although we acknowledge that these considerations are likely to be important in reality, the mechanism we highlight would also underlie diffusion in a social network with a stable, nonregular topological structure - although it would be considerably more difficult to characterize in that case (see the discussion in Section 6).
} 
$\bar{q}>\underline{q}$, which measures units of quality-adjusted provision for each dollar's worth of provision through provider $j{ }^{3}$ Thus, given each individual $i$ 's contribution, $v_{i, t}$, to her selected provider at, $j(i, t)$, at $t$, and given quality realizations, $q_{j, t}$, the effective level of collective consumtpion experienced by all individuals at $t$ will equal $G_{t}=\sum_{i} q_{j(i, t), t} v_{i, t}$. Without loss of generality, we assume $\bar{q}=1$ and $\underline{q}=0$.

The quality of provision of provider $j$ at time $t$ is ex-ante unobservable but is observable ex post to individuals who have made positive contributions to provider $j$ at $t .{ }^{4}$ Quality of provision for provider $j$ evolves stochastically over time, according to the following conditional distribution: $\operatorname{Pr}\left(q_{j, t}=\underline{q} \mid q_{j, t-1}=q\right)=\operatorname{Pr}\left(q_{j, t}=\right.$ $\left.\bar{q} \mid q_{j, t-1}=\bar{q}\right)=\rho>1 / 2$; i.e. quality remains the same from one period to the next with probability $\rho>1 / 2$ and changes with probability $1-\rho$. Any given individual can only identify provider $j$ if she has contributed to it at $t-1$. This means that the only portion of $i$ 's history that determines her information set at $t$ is the pair $\left(j(i, t-1), q_{j(i, t-1), t-1}\right)$, where $j(i, t)$ denotes the provider selected by $i$ in period $t$. As we are concerned here with transmission of private information on provision quality, we can abstract from other determinants of quality that non-stochastic or public information. ${ }^{5}$

We assume that quality evolves independently across providers. Thus, with $M$ large, and given our assumption of symmetric transition probabilities, the propor-

\footnotetext{
${ }^{3}$ Heterogeneity with respect to quality could equivalently be modelled in terms of unobservable marginal costs.

${ }^{4}$ In general, one would expect that, even if individuals that direct contributions towards a certain provider are not able to perfectly observe quality, they still receive more information about quality than individuals who do make contributions towards that provider. Our assumption represents an extreme case that is presentationally convenient.

${ }^{5}$ In reality, quality does not just evolve stochastically and so there may be persistent heterogeneity across providers, which would ultimately become public information; but there are also new varieties being introduced and new providers of unknown quality, which implies that information about providers depreciates and new learning occurs.
} 
tion of quality types in the population will converge through time to $1 / 2$ for each type.

All individuals have identical, risk-neutral preferences for private and collective consumption within each period. These are represented by

$$
U\left(x_{i, t}, G_{t}, e_{i, t}\right)=x_{i, t}+\theta G_{t}-e_{i, t},
$$

where $e_{i, t}$ is private effort directed towards information sharing (see below). Individuals have disposable income $y_{i, t}=y=1+\mu(\mu>0)$ in all periods. Consumption is bounded below to unity and therefore contributions must lie between zero and $\mu$. We assume $2>\theta>1 / \rho$ (the role of this assumption is explained below). In this specification, individuals only care about their own contributions towards $G_{t}$ and those of others because of a consumption motive; in Section 4, we discuss how our analysis and results carry over to a setting where individuals are motivated by warm-glow effects.

Within each period, $t$, nature assigns each individual $i \in I$ a social neighbourhood, $S_{i, t}$, consisting of $b$ individuals (excluding the individual herself), where $1 \leq b<N$. Neighbourhoods can be overlapping, but the structure of social neighbourhoods is such that each individual has exactly $b$ neighbours. The $b$ individuals that form $i$ 's neighbourhood at $t$ are newly sampled at random from the population of $N$ individuals in each period. ${ }^{6}$

In each period $t$, individuals can choose to inform each of their neighbours, $i^{\prime} \in S_{i, t}$, of the quality they experienced from provider $j(i, t-1)$, sending each a signal $\sigma_{i, i^{\prime}, t}=(j, q)$, with $j=j(i, t-1)$. If they do so, they incur a cost $c$ (translating into a higher $e_{i, j}$ in (1)) for each neighbour they inform. A signal is truthful if the

\footnotetext{
${ }^{6}$ The resulting social network is representable by a regular graph of degree $b$, randomly sampled in each period from the set of all possible regular graphs of degree $b$.
} 
quality level reported corresponds to the quality actually experienced by $i$, i.e. if it reports $q=q_{j(i, t-1), t-1}$.

Individuals are indefinitely lived and myopic. This means that choices with respect to sending costly signals in any given period only account for how those signals may affect provision in that period (the implications of forward-looking signalling choices are briefly discussed in the concluding section).

\section{Sequence of actions and events}

The sequence of actions and information sets in each time period $t$ are as follows: (i) at the beginning of each time period $t$, nature generates social neighbourhoods, $S_{i, t}$, and updates providers' quality types, $q_{j, t}$; (ii) each individual $i \in I$ for whom $v_{i, t-1}>0$ is fully informed about $q_{j(i, t-1), t-1}$, and can choose to send signals $\sigma_{i, i^{\prime}, t}, i^{\prime} \in S_{i, t}$ simultaneously with the signaling choices of other individuals; (iii) each individual, $i$, receives signals $\sigma_{i^{\prime}, i, t}$ from her neighbours, $i^{\prime} \in S_{i, t}$, and updates her information set; (iv) each individual $i \in I$ selects a provider, $j(i, t)$; (v) once they have selected a provider, individuals then simultaneously make contributions, $v_{i, t}$, to providers $j(i, t) ;(\mathrm{vi})$ individuals who have made positive contributions $\left(v_{i, t}>0\right)$ observe provision qualities, $q_{j(i, t), t}$, and everyone experiences collective consumption $G_{t} \cdot{ }^{7}$

We will discuss within period actions starting from contribution choices (v) and then moving backwards to provider selection choices (iv), and signaling choices (ii).

\footnotetext{
${ }^{7}$ This means that average quality is observable to everyone, but only individuals who have made contributions are able to link quality of provision of their chosen supplier to its identity.
} 


\section{Contribution choices}

Suppose individual $i$ has selected provider $j(i, t)=j^{\prime}$, and let $E_{i}\left[q_{j^{\prime}, t}\right] \equiv \tilde{q}$ be $i^{\prime}$ s expected level of quality from provider $j^{\prime}$ given $i$ 's information. Expected quality can take one of three values, depending on the information $i$ has. If $j^{\prime}$ is a provider from whom $i$ has experienced high quality at $t-1$, or if it is a provider that one of $i$ 's neighbours has signaled as being of high quality at $t-1$, then $\tilde{q}=\rho>1 / 2$; if it is a randomly selected provider, then, for $t$ large enough (implying that the proportions of suppliers of each quality type are the same types for both types), expected quality is $\tilde{q}=1 / 2$; if it is a provider from whom $i$ has experienced low quality at $t-1$, expected quality is $\tilde{q}=1-\rho<1 / 2$.

Constrained utility maximization in period $t$ then gives $v_{i, t}=0$ or $v_{i, t}=\mu$ depending on whether $\tilde{q}$ is less than or greater than $1 / \theta$. Given our earlier assumption that $2>\theta>1 / \rho($ or $\rho>1 / \theta>1 / 2)$, contributions will be $v_{i, t}=\mu$ for $\tilde{q}=\rho$ and $v_{i, t}=0$ otherwise, i.e. individuals only make positive contributions if the expected quality of provision from their selected provider exceeds average quality, $1 / 2$.

\section{Selection of providers}

Consider first individuals who have experienced high quality from their chosen provider at $t-1, j(i, t-1)$. If they receive no additional information, then, given that $\rho>1 / 2$, they would elect to go back to the same provider - as doing so yields a higher expected quality than selecting a new supplier at random. If they are tipped off by a neighbour concerning a supplier that has delivered high quality at $t-1$, and they believe the information to be truthful, they are indifferent between switching to this alternative supplier and sticking to the supplier selected at $j(i, t-$ 1).

Consider next individuals who have experienced low quality from their chosen 
provider at $t-1$. If they receive no additional information, given that $1-\rho<1 / 2$, they would elect to select a new supplier - since doing so yields a higher expected quality than going back to the same provider. If they are tipped off by a neighbour about a supplier that has delivered high quality at $t-1$, i.e. if they receive a signal, $\sigma_{i^{\prime}, i, t}=\left(j^{\prime}, q^{\prime}\right)$ from one of their neighbours, they must choose between acting on the advice received or selecting a new supplier at random. As long as they take any signal received to be truthful (a question that we shall examine below), i.e. if $j^{\prime}=j(i, t-1)$ and $q^{\prime}=q_{j(i, t-1), t-1}$, selecting $j^{\prime}$ will deliver a higher expected quality than selecting a supplier at random; so they will select $j^{\prime}$.

If an individual simultaneously receives multiple signals from different neighbours, then, as long as those signals can be taken to be truthful, it is irrelevant which particular signal the receiver will act upon. Thus we will assume that one signal is selected at random. This is without loss of generality: as we shall show, the equilibria we analyze only feature truthful signals, implying that the choice of tie-breaking rule is immaterial.

\section{Information sharing choices}

An individual who has experienced high quality from her chosen supplier at $t-1$ may be willing to incur a cost - reflected in (1) via the effort term, $e_{i, t}$ - to share information about her provision experience with her neighbours because she will benefit from the effect that better information has on her neighbours' contribution choices. Specifically, if any given one of $i^{\prime}$ s neighbours, $i^{\prime} \in S_{i, t}$, has no information, her contribution will be $v_{i^{\prime}, t}=0$, whereas, if $i^{\prime}$ has information about a supplier that has delivered high quality at $t-1$, then she will make a direct a contribution $v_{i^{\prime}, t}=\mu$ towards that supplier, resulting in a higher payoff for $i$. Denoting by $E\left[G_{t}^{-i^{\prime}}\right]$ the expected, quality-adjusted level of provision from the contributions of all individuals other than $i^{\prime}$, the public good-related component of the payoff 
experienced by $i$ when $i^{\prime}$ is uninformed is therefore $\theta E\left[G_{t}^{-i^{\prime}}\right] \equiv \Phi$; when $i^{\prime}$ is informed, the corresponding level is $\theta\left(E\left[G_{t}^{-i^{\prime}}\right]+\rho \mu\right) \equiv \bar{\Phi}=\underline{\Phi}+\theta \rho \mu>\underline{\Phi}$. So, if the effort cost, $c$, of sending an informative signal to her neighbours is not too high relative to the expected gain $\theta \rho \mu \equiv \Psi, i$ may choose to voluntarily incur that cost. We can interpret this behaviour as fundraising. Note that in an analogous setting where private information about supplier quality concerns private consumption, individuals would never incur a private cost to inform their neighbours about their own consumption experience. It is only in the case of contributions to collective consumption that the actions of uninformed individuals are of concern to better informed individuals, inducing them to actively share their information with others. $^{8}$

In the calculation of whether or not to send a signal to her neighbours, each informed individual must also consider the likelihood that her signal will make a difference for the neighbour who receives it. As discussed above, any neighbour receiving the signal will only find it valuable if she has experienced low quality in period $t-1$ and is therefore uninformed. Also, the signal will only be valuable if the uninformed neighbour does not also receive another signal from another neighbour. ${ }^{9}$ Thus, information sharing decisions involve both an assessment of the likelihood that neighbouring individuals are uninformed and of the likelihood that they might also be targeted by other fundraisers.

Let $k_{t}$ be the proportion of individuals in the population who, having experienced high quality at $t-1$, are able to identify a provider who was a high-quality

\footnotetext{
${ }^{8}$ An individual, $i$, could in principle also choose to send signals about a provider $j(i, t-1)$ from whom she has experienced low quality in order to prevent neighbours from selecting $j(i, t-1)$. However, as long as $M$ is sufficiently large, the probability of a neighbour selecting $j(i, t-1)$ at random is negligible, and so $i$ will never have an incentive to incur a $\operatorname{cost} c$ to send a signal in this case.

${ }^{9}$ In this setup, additional informative signals do not improve information.
} 
supplier in period $t-1$, i.e. the proportion of individuals that have no need of further information. Assume for now that $k_{t}$ is publicly known. Then, given that an individual's neighbours are a random sample of the whole population, the probability that each neighbour will be able to make use of an informative signal is $1-k_{t}$, and the probability that each of her $b$ neighbours will be informed - and therefore will be in a position to send the signal herself - is $k_{t}$. Suppose then that all informed individuals other than $i$ send a signal at the beginning of period $t$ with probability $s_{-i, t}$. From the point of view of an informed individual, $i$, the probability that any given neighbour will benefit from her signal is equal to the probability of the neighbour finding any signal valuable - which equals $1-k_{t}$ - times the probability that this neighbour will not receive a signal from an informed neighbour other than $i$ - which equals $\left(1-k_{t} s_{i, t}\right)^{b-1}$. So, the expected net value to $i$ of sending a signal to one of her neighbours is

$$
\left(1-k_{t}\right)\left(1-k_{t} s_{-i, t}\right)^{b-1} \Psi-c \equiv \Lambda\left(s_{-i, t}, k_{t}\right)
$$

An individual $i$ will then always send a signal $\left(s_{i, t}=1\right)$ if $\Lambda\left(s_{-i, t}, k_{t}\right)>0$, will never do so $\left(s_{i, t}=0\right)$ if $\Lambda\left(s_{-i, t}, k_{t}\right)<0$, and will be indifferent between sending and not sending a signal $\left(0<s_{i, t}<1\right)$ if $\Lambda\left(s_{-i, t}, k_{t}\right)=0$.

All of this presumes that individuals only send truthful signals. But an individual might also have an incentive to send an untruthful signal, i.e. to signal high quality for a provider that has delivered low quality. This is because, given that all individuals benefit from the provision of collective consumption, and given that the contribution level selected by an uninformed individual is zero, deceitfully inducing an uninformed individual to make a positive contribution to a randomly selected provider of expected quality $1 / 2$ yields an expected public good-related benefit $\theta \mu / 2>0$. Nevertheless, we will show below that untruthful signals can be ruled out in a strictly mixed-strategy equilibrium signaling choices. 


\section{Information sharing, information diffusion, and neighbourhood size}

We next move to the question of how information sharing and private provision are affected by neighbourhood size, $b$. For this purpose, we first characterize equilibria in signaling and contribution choices in any given period (for a given $k_{t}$ ), and then derive stationary state conditions for an equilibrium of the dynamic game (with $k_{t}$ endogenous).

\subsection{Within-period equilibria}

\section{Equilibrium information sharing choices}

We want to study how neighbourhood size affects information sharing effort, $s_{i, t}$, on the intensive margin, i.e. for $s_{i, t}$ strictly between zero and unity. For a given, publicly known $k_{t}$, a symmetric equilibrium in signaling choices with $s_{i, t}=s_{-i, t}=$ $s_{t}, 0<s_{t}<1$ (giving rise to contribution choices as described in the previous section) requires $\Lambda\left(s_{t}, k_{t}\right)=0$. A corner (a pure-strategy equilibrium) where $s_{t}=0$ can be ruled out as long as signaling costs, $c$, are not too large; and a corner where $s_{t}=1$ can be ruled out as long as signaling costs, $c$, are not too small. Setting (2) equal to zero and solving for $s_{t}$, we obtain

$$
s_{t}=\frac{1}{k_{t}}\left(1-\left(\frac{c}{\left(1-k_{t}\right) \Psi}\right)^{1 /(b-1)}\right)
$$

One can verify that this is strictly between zero and unity if and only if $1-k_{t}>$ $c / \Psi>\left(1-k_{t}\right)^{b}$.

We next show that untruthful signals can be ruled out in an interior equilibrium. Suppose that an untruthful signal were sent when everyone else is sending truthful signals. Then, starting from the belief that the signals that are sent 
are truthful, an uninformed individual receiving a single signal would act on it; an uninformed individual receiving multiple signals would have to decide which particular signal to act upon. Earlier, we have stated the assumption that, when multiple signals are received, one particular signal will be selected at random (with equal probability as any other signal); but we can generalize this tie-breaking rule and simply posit that, if a signal is sent, and the receiver believes signals to be truthful, then that signal will be selected and acted upon with a probability that is either positive or zero. Then, assuming that all informed individuals other than $i$ are sending truthful signals, the expected net value to $i$ of sending an untruthful signal to one of her neighbours is

$$
\left(1-k_{t}\right)\left(1-k_{t} s_{t}\right)^{b-1} \theta \mu / 2+\mathrm{Y}-c
$$

where the first term represents the expected gain associated with the possibility that an individual not receiving a signal from anyone else would act on the untruthful signal and $Y \leq 0$ is the expected loss associated with the possibility that an individual receiving multiple signals selects $i$ 's untruthful signal over the truthful signal of another neighbour, resulting in a lower expected level of quality-adjusted provision. ${ }^{10}$ In a strictly mixed-strategy equilibrium where $\Lambda\left(s_{t}, k_{t}\right)=0$, the signaling cost, $c$, must equal $\left(1-k_{t}\right)\left(1-k_{t} s_{t}\right)^{b-1} \theta \mu \rho$; and, since $\theta \mu / 2<\theta \mu \rho=\Psi$, and $Y \leq 0$, we can conclude that expression (4) must be negative. This implies that an outcome where only truthful signals are sent, and where receivers have no reason to doubt the signals' truthfulness, is an equilibrium outcome. ${ }^{11}$

\footnotetext{
${ }^{10}$ If sending an untruthful signal when all other signals are truthful generates a non-zero probability of a receiver selecting a provider $j$ for whom $q_{j, t-1}=\underline{q}$, then $\mathrm{Y}$ is strictly negative, otherwise it is zero.

${ }^{11}$ Since signals are costly to send, an outcome where some all signals are untruthful and are disregarded cannot be an equilibrium outcome - if signals are disregarded, then senders would opt to send no signals and save the cost. An outcome where some signals are truthful and others are
} 


\section{Information sharing and neighbourhood size}

We can rearrange condition (3) for a symmetric equilibrium in strictly mixed strategies and express it as

$$
k_{t} s_{t}=1-\left(\frac{c}{\left(1-k_{t}\right) \Psi}\right)^{1 /(b-1)}
$$

The left-hand side of (5) is a measure of information sharing intensity in the economy - information sharing per head per neighbour. This is negatively related to signaling costs, $c$. We will denote information sharing intensity as $r_{t} \equiv k_{t} s_{t}$. Trivially, for a given $s_{t}$, a higher $k_{t}$ results in a higher $r_{t}$; but $r_{t}$, taken as a whole, varies with $k_{t}$ as described by the right-hand side of (5):

Proposition 1 Information sharing intensity, $r_{t}=k_{t} s_{t}$, is negatively related to the stock of information, $k_{t}$, and to neighbourhood size, $b$.

PROOF: Denote the right-hand side of (5) with $\Omega\left(k_{t}, b\right)$. Differentiating this with respect to $k_{t}$ and $b$, and noting that $\Lambda\left(s_{t}, k_{t}\right)=0$ implies $c /\left(\left(1-k_{t}\right) \Psi\right)=\left(1-r_{t}\right)^{b-1}<1$, we obtain, after substitution,

$$
\Omega_{k_{t}}=-\frac{1-r_{t}}{(b-1)\left(1-k_{t}\right)}<0
$$

and

$$
\Omega_{b}=\frac{1-r_{t}}{(b-1)^{2}} \ln \left(\left(1-r_{t}\right)^{b-1}\right)<0
$$

The result that $r_{t}$ decreases in $b$ for a given $k_{t}$, also implies that $s_{t}$ decreases with $b$.

not can also be ruled out by analogous arguments - i.e. given any mix of truthful and untruthful signals, and receivers' beliefs consistent with that mix, then withholding an untruthful signal is strictly payoff-improving for the sender. 
The intuition for this result is straightforward. Although private contributions are made towards provision of a pure public good, information sharing with one's neighbours has the characteristics of a local public good. To be precise, it is a local form of provision that is only indirectly purely public: an individual can only receive signals from her neighbours, and so provision of information to neighbours is in itself not a pure public good despite the fact that its benefits ultimately flow through the provision of a pure public good. Since only neighbours can provide information to an uninformed individual, free-riding incentives with respect to information sharing remain contained within a given neighbourhood. The larger an individual's social neighbourhood, then, the greater the free-riding incentives in information sharing.

\subsection{Information diffusion}

\section{Information dynamics}

Proposition 1 deals with the relationship between neighbourhood size and signaling choices for a given stock of information, $k_{t}$. However, in a multi-period economy where individuals make repeated signaling and contributions choices as detailed in Section 2 - the stock of information is endogenous. In what follows, we look at the relationship between neighbourhood size and information sharing when the endogeneity of $k_{t}$ is accounted for.

The stock of information, $k_{t}$, evolves through time as follows:

$$
k_{t+1}=k_{t} \rho+\left(1-k_{t}\right)\left(1-\left(1-k_{t} s_{t}\right)^{b}\right) \rho \equiv \Gamma\left(s_{t}, k_{t}\right) .
$$

The term $k_{t} \rho$ in (8) represents the fraction of individuals who, having experienced high quality at $t-1$, do a repeat purchase at $t$ and again experience high quality at $t$; the next term represents the fraction of individuals who, having experi- 
enced low quality at $t-1$, receive an informative signal at $t$ (which occurs with probability $\left.1-\left(1-k_{t} s_{t}\right)^{b}\right)$, and, having acted on it, experience high quality; since only informed individuals participate in provision (whether they have personally gathered information or they have received signals from neighbours), no new information will be gained by uninformed individuals who receive no signals from neighbours.

Up to this point, we have simply assumed that the proportion of informed individuals, $k_{t}$, is publicly known. But given that quality realizations are only observable by contributors, $k_{t}$ is not directly observable. However, since in a large economy the realized level of public good provision, $G_{t}$ equals $\rho k_{t}$ with probability one, and since individuals experience (and thus observe) the level of public good provision, they can perfectly infer $k_{t}$ from $G_{t}$ at the end of period $t$ (i.e. after signaling and contribution choices for period $t$ have been made). On the basis of their knowledge of $k_{t}$, individuals can then determine the level of $k_{t+1}$ from (8) before making their choices at $t+1$. This amounts to $k_{t}$ being publicly observable at the beginning of each period.

In this setting, knowledge of good suppliers spreads to others - at a speed that depends on the size of social neighbourhoods and the cost of information transmission. But information always remains incomplete due to the fact that suppliers undergo quality shocks; i.e. there is social learning, but it never leads to information being complete as information "depreciates".

\section{Stationary states}

A stationary state consists of an indefinite sequence of periods where information sharing choices are in equilibrium within each period given the amount of information about high-quality providers in the economy, and where the amount of information stays the same through time, i.e. where $k_{t+1}=k_{t}=\hat{k}$. 
A stationary state can thus be characterized with reference to a fixed point, $(\hat{s}, \hat{k})$, such that

$$
\Lambda(\hat{s}, \hat{k})=0,
$$

and

$$
\hat{k}=\Gamma(\hat{s}, \hat{k}) .
$$

This definition implicitly incorporates a condition on the consistent updating of beliefs about $k_{t}$, as previously discussed. ${ }^{12}$

Focusing on (9) and (10), we can totally differentiate the equilibrium conditions with respect to $\hat{r} \equiv \hat{k} \hat{s}$ and $b$ in order to derive an expression for the total derivative $\mathrm{d} \hat{r} / \mathrm{d} b$. This is unambiguously negative:

Proposition 2 In a stationary state, information sharing intensity, $\hat{r}=\hat{k} \hat{s}$, is negatively related to neighbourhood size, $b$.

\footnotetext{
${ }^{12}$ We abstract from questions of global convergence (Gale and Kariv, 2003, derive global convergence results for a setup that is somewhat related to ours but does not share the same formal structure). We can nevertheless readily derive local convergence (stability) results. Suppose that, starting from stationary state with $k_{t+1}=k_{t}=\hat{k}$, in a given period, $s$, the stock of information undergoes an exogenous shock, $\epsilon$, becoming $k_{s}=\hat{k}+\epsilon$. Then, for $\epsilon \rightarrow 0$, a sufficient condition for subsequent convergence back to $\hat{k}$ is $\left|\Gamma_{k}\right|<1$. Substituting the expression for $\Omega(\hat{k}, b)$ (the righthand side of (5)) into $\Gamma(\hat{s}, \hat{k})$ (expression (8)), and differentiating with respect to $\hat{k}$, we obtain

$$
\Gamma_{\hat{k}}=-\frac{\rho(c /(\Psi(1-\hat{k})))^{b /(b-1)}}{b-1} .
$$

Noting that $0<(c /(\Psi(1-\hat{k})))^{1 /(b-1)}<1($ from (5)), $0<\rho<1$, and $0<1 /(b-1)<1$, we conclude that $\left|\Gamma_{k}\right|<1$.
} 
PROOF: Solving for $\hat{k}$ from (10) as a function of $\hat{r}$, we obtain

$$
\hat{k}=\rho \frac{1-(1-\hat{r})^{b}}{1-\rho(1-\hat{r})^{b}} \equiv \Theta(\hat{r}, b)
$$

Expressing (9) as a function of $\hat{k}$ and $\hat{r}$, i.e. as $\tilde{\Lambda}(\hat{r}, \hat{k}) \equiv(1-\hat{k})(1-\hat{r})^{b-1}-c=0$, substituting (12) into it, and totally differentiating, we obtain

$$
\frac{\mathrm{d} \hat{r}}{\mathrm{~d} b}=-\frac{\tilde{\Lambda}_{\hat{k}} \Theta_{b}+\tilde{\Lambda}_{b}}{\tilde{\Lambda}_{\hat{k}} \Theta_{\hat{r}}+\tilde{\Lambda}_{\hat{r}}}=\frac{(1-\hat{r}) \ln (1-\hat{r})}{b-1+\rho(1-r)^{b}}<0 .
$$

The conclusion that a larger neighbourhood size results in lower information sharing intensity thus also applies to comparisons across steady states that take into into account information dynamics.

Note that the above result does not immediately imply that collective consumption is negatively affected by an increase in neighbourhood size. The total expected surplus associated with collective consumption - gross of signaling costs increases with the information stock $\hat{k}$. As shown earlier, for a given $b$, the stock of information is directly related to information sharing intensity, $\hat{r}$; and, as just shown, information sharing intensity decreases with $b$. However a larger $b$ also has a direct positive effect of information diffusion - the expression $\Theta_{b}$ is positive (see proof below). In principle, if this latter effect were to dominate the former, information on supplier quality could, in a stationary equilibrium, be positively related to neighbourhood size even if it is associated with a lower level of information sharing intensity. It can be shown that this can never be the case.

Proposition 3 In a stationary state, the stock of information, $\hat{k}$, about supplier quality, as well as expected, quality-adjusted provision, $E[G]$, are decreasing in the size of social neighbourhoods, $b$. 
PROOF: The total effect of an increase in $b$ on $\hat{k}$ is expressed by the total derivative

$$
\frac{\mathrm{d} \hat{k}}{\mathrm{~d} b}=\Theta_{b}+\Theta_{\hat{r}} \frac{\mathrm{d} \hat{r}}{\mathrm{~d} b}
$$

We have

$$
\begin{aligned}
& \Theta_{\hat{r}}=\frac{\rho(1-\rho)(1-\hat{r})^{b-1} b}{\left(1-\rho(1-\hat{r})^{b}\right)^{2}}>0 ; \\
& \Theta_{\hat{b}}=-\frac{\rho(1-\rho)(1-\hat{r})^{b-1} \ln (1-\hat{r})}{\left(1-\rho(1-\hat{r})^{b}\right)^{2}}>0 .
\end{aligned}
$$

We can substitute these into (14) in conjunction with the expression for $\mathrm{d} \hat{r} / \mathrm{d} b$. After simplification, we obtain

$$
\frac{\mathrm{d} \hat{k}}{\mathrm{~d} b}=\frac{\rho(1-\rho)(1-\hat{r})^{b} \ln (1-\hat{r})}{\left(1-\rho(1-\hat{r})^{b}\right)\left(b-1+\rho(1-\hat{r})^{b}\right)}<0
$$

Since expected collective provision at $t$ equals $k_{t+1} \mu$, which in turn equals $k_{t} \mu$ in a stationary equilibrium, we can also conclude that an increase in neighbourhood size decreases expected provision of the collective good.

Thus, larger social neighbourhoods unambiguously result in a lower level of expected collective consumption - although this remains strictly positive as long as some signaling takes place (i.e. as long as $\hat{s}>0) .{ }^{13}$

These result can be illustrated by the following parameterized example. Consider a scenario with $\rho=2 / 3, \theta=7 / 4>1 / \rho, \mu=1 / 2, c=\Psi / 2=7 / 24$. For $b=2$, we have $\hat{s} \simeq 0.45, \hat{k} \simeq 0.39, \hat{r}=\hat{s} \hat{k} \simeq 0.18, E[\hat{G}] / N \simeq 0.195$. Doubling neighbourhood size to $b=4$ results in $\hat{s} \simeq 0.22, \hat{k} \simeq 0.36, \hat{r} \simeq 0.08, E[\hat{G}] / N \simeq 0.18$.

\footnotetext{
${ }^{13}$ If signaling goes to zero, only individuals who experience high quality in a given period will engage in provision in the next period, whereas those who do not will permanently cease to contribute - implying that both the stock of information and the level of provision will decline to zero.
} 
Finally, for $b=8$, we have $\hat{s} \simeq 0.11, \hat{k} \simeq 0.345, \hat{r} \simeq 0.04, E[\hat{G}] / N \simeq 0.17 .{ }^{14}$

Collective consumption is lower when social neighbourhoods are larger even if, in this setup, the spillovers from collective provision are independent of neighbourhood size. Social neighbourhoods are only relevant here for voluntary information sharing. An increase in neighbourhood size increases free-riding incentives in information sharing, reducing information diffusion about provider quality and thus expected provision, despite the potential for a higher number of signals reaching any given individual in larger neighbourhoods.

\section{Extensions}

So far the analysis has been carried out in a model where private contributions come about purely from consumption motives that are perfectly aligned across individuals. In this section we show how our analysis and results extend to alternative characterizations of the motives underlying giving and information sharing behaviour.

\subsection{Warm-glow motives}

In the linear specification of preferences we have assumed, although the public good is a pure public good, the choice of whether or not to contribute is structurally independent of the level of contributions by others, i.e. donations by one individual do not crowd out donations by others. This also implies that individual donations are independent of group size. Our arguments, however, do not crucially hinge on ruling out free riding; we are simply modeling situations where individuals have positive incentives to give (as we observe them to do), given the

\footnotetext{
${ }^{14}$ One can verify that in all scenarios, the condition $1-\hat{k}<c / \Psi<(1-\hat{k})^{b}$ for an interior equilibrium with $0<\hat{s}<1$ is satisfied.
} 
contribution choices of others, and where acquiring information can affect such incentives. In fact, our specification is fully equivalent to one where preferences are strictly convex - implying that, in principle, free riding can occur-consumption is bounded below to unity, and the marginal rate of substitution of private to public consumption $\left(U_{G} / U_{x}\right)$ is greater than $\rho$ for $\left(x_{i, t}=1, G_{t}=N \mu\right)$, and is less than $1 / 2$ for $\left(x_{i, t}=1+\mu, G_{t}=0\right)$ - which means that the individual will select a donation $v_{i, t}=\mu$ if $\tilde{q} \geq \rho$ and a donation $v_{i, t}=0$ if $\tilde{q} \leq 1 / 2$. Thus, our arguments only require that individuals make positive donations (conditional on the information they hold) in the "region of interest".

Nevertheless, with strictly convex preferences and in the absence of any further restrictions, private giving will tend to vanish as the number of donors gets larger. In order to rationalize non-vanishing contributions in large economies, economists have hypothesized that individuals may also experience a private benefit ("warm glow") from their donations (Andreoni, 1990). In the following, we show that the above analysis and results are robust with respect to the inclusion of warm-glow motives.

If warm glow only relates to the donation an individual makes, then it would simply involve an additional, private benefit term, $\gamma g_{i, t}$, with $g_{i, t} \equiv q_{j, t} v_{i, t}$, in (1), which would lower the minimum level of the marginal rate of substitution between public and private consumption (represented by $\theta$ in our linear specification) that is required to rationalize positive donations. The rest of the model and analysis would be qualitatively unaffected. On the other hand, if warm glow only arises from the donation individuals make and is their only reason for giving, i.e. if $\gamma>0$ and $\theta=0$, then there would exist no individual incentive to engage in costly signaling.

However, it seems plausible to conjecture that individuals who derive warm glow from their own direct contributions to collective consumption would also derive warm glow from any positive effect on collective consumption that they may 
be able to bring about through their own information sharing efforts, i.e. from other individuals' contributions for which they can "take credit". Then, if an individual sends an informative signal of quality to a neighbour, she would also experience warm glow from that neighbour's contributions in those realizations where the signal is valuable to the neighbour and affects her giving choices.

This idea can be modelled as follows. Let $\sigma^{0}$ denote the empty signal, $\mathcal{E}\left(s_{i, t}, i \in\right.$ I) denote the event space given the mixed signaling strategies chosen by individuals, and let $\mathcal{E}_{i, h}\left(s_{i, t}, i \in I\right)$ be the subset of realizations where $\sigma_{i, h, t} \neq \sigma^{0}$ and $\sigma_{i^{\prime}, h, t}=\sigma^{0}, i^{\prime} \in S(h), i^{\prime} \neq i$, i.e. those realizations where individual $h$ receives a signal from $i$ and not from anyone else in her social neighbourhood. Suppose the payoff of individual $i$ in a given realization $\varepsilon \in \mathcal{E}\left(s_{i, t}, i \in I\right)$ is

$$
x_{i, t}+\gamma\left(g_{i, t}+\sum_{h \in\left\{h \in S(i) \mid \varepsilon \in \mathcal{E}_{i, h}\right\}} g_{h, t}\right)
$$

i.e., the individual puts a marginal valuation of $\gamma$ on her own quality-adjusted donations as well as on the donations of those neighbours for whom her signaling efforts have "made a difference".

In this case an informed individual's expected payoff from sending a signal to a neighbour is equal to the probability that the neighbour is uninformed times the probability that the neighbour has not received a signal from someone else, times $\gamma \rho \mu$, minus the cost of signaling, $c$; i.e. an expression that is identical to $\Lambda\left(s_{t}, k_{t}\right)$, as defined in (2), but for the fact that the scalar $\theta$ is now replaced by $\gamma$. The analysis of information sharing equilibria can then proceed along the same lines as in the case where giving stems purely from consumption motives, and the results and 
conclusions are also identical. ${ }^{15}$

The only difference between this alternative characterization and the one developed in the previous sections lies in the interpretation of the neighbourhood size effect. When the benefits from information sharing stem from a consumption motive, information sharing can be thought of as a local public good; accordingly, an increase in the size of social neighbourhoods induces free riding in the provision of this public good - the larger the size of social neighbourhoods, the more individuals can rely on others to inform their neighbours. When the benefits from information sharing stem from a warm-glow motive, on the other hand, information sharing can be thought of as a private good subject to local congestion; in this case an increase in the size of social neighbourhoods directly reduces the warmglow value of information sharing - the larger the size of social neighbourhoods, the more difficult it is for an individual who send an informative signal to take credit for other individuals' contributions. The formal analysis of the problem remains exactly the same.

\subsection{Heterogeneous preferences for collective consumption}

An important dimension of information sharing activities that is absent from our previous analysis is the potential for information sharing efforts to steer other people's charitable activities towards goals that the fundraiser favours; i.e. individuals who engage in fundraising do not just do so in order to share information with others, but also hope to have an effect on the kind of charitable activities that other

\footnotetext{
${ }^{15}$ Note that this also applies to the conclusion that only truthful signals will be sent in a mixedstrategy equilibrium: even if an untruthful signal can generate a warm-glow benefit, since sending a truthful signal generates a larger benefit, in an equilibrium where the net expected benefit of sending a truthful signal is zero, the net expected benefit of sending an untruthful signal is negative, and so no untruthful signal will be sent.
} 
individuals support.

We can incorporate this idea into our analysis by allowing for preference heterogeneity with respect to collective consumption. Suppose that there are two forms of collective provision, 1 and 2, and that individuals have heterogeneous preferences with respect to the two forms of provision; namely, half of the population (type 1) have a period-t payoff equal to

$$
x_{i, t}+2 \theta\left(\alpha G_{t}^{1}+(1-\alpha) G_{t}^{2}\right)-e_{i, t}
$$

whereas the other half (type 2) have a payoff equal to

$$
x_{i, t}+2 \theta\left((1-\alpha) G_{t}^{1}+\alpha G_{t}^{2}\right)-e_{i, t}
$$

with $\alpha>1 / 2$ - meaning that, quality being constant, the first half would prefer to contribute towards collective good 1 and the other half would would prefer to contribute towards collective good 2. In addition assume that $\rho(1-\alpha) \bar{q}>(1 / 2) \alpha \underline{q}$, implying that an individual of a given type would contribute towards the collective good she does not favour through a known high-quality supplier rather than contribute towards the good she favours through a supplier of unknown quality. ${ }^{16}$

Then, an individual, $i$, of a given type who has information about quality for a supplier providing the collective good she favours would face even stronger incentives to engage in costly signaling, because information sharing increases the chance that an uninformed neighbour, $i^{\prime}$, of a different type would opt to contribute to the good she (individual $i$ ) favours - if the only signal $i^{\prime}$ receives is $i^{\prime}$ s signal; and it would also increase the chance that an individual of the same type

\footnotetext{
${ }^{16}$ When this condition is not met, the only individual interactions that are relevant are those between individuals belonging to the same preference type - a scenario that has the same structure and properties of a single-good scenario.
} 
as $i$ 's type who is only informed about provision of the good she does not favour would switch to her more favoured good - if she receives a signal from $i$. In other words, a fundraiser can be pivotal in her neighbours' choice of which form of collective provision to support.

In addition, if individuals who are informed about the good that they favour engage in information sharing with a positive probability that is less than unity (i.e. in an interior mixed-strategy outcome), individuals who have information about the good they do not favour will not do so - since the expected gain from sending a signal in this case is strictly less than for individuals who have information about they favoured good. Thus, a smaller proportion of informed individuals will engage in information sharing, which will in turn increase signaling incentives for those who do so.

As a result, information sharing intensity and the overall level of collective provision will rise. This result is formalized in the proof of the following proposition.

Proposition 4 In a stationary equilibrium, the stock of information on supplier quality, and the level of expected, quality-adjusted provision, $E\left[\hat{G}^{1}+\hat{G}^{2}\right]$, are increasing in $\alpha$ ( $\alpha \geq$ $1 / 2)$.

Proof: Consider the signaling incentives for an individual of type 1 . Let $\Lambda_{t}^{\tau \tau}$ be the expected net payoff in period $t$ of an individual of type $\tau(\tau \in\{1,2\})$ for sending a signal about quality of the good she favours, and $\Lambda_{t}^{\tau \tau^{\prime}}\left(\tau^{\prime} \neq \tau\right)$ be the corresponding payoff for a signal about the good she does not favour. Because the good she favours is valued more, it must be the case that if $\Lambda_{t}^{\tau \tau}=0$, then $\Lambda_{t}^{\tau \tau^{\prime}}<0$, and so in a mixed-strategy equilibrium individuals will only send truthful signals of quality about the good they favour (we state this conclusion informally for the time being, but we verify it formally later on in our proof). The expected payoff for an informed individual of type 1 from sending a truthful signal about provision of good 1, if the only other signals are truthful signals about good 2 
sent by informed individuals of type 2 , is then

$$
\begin{aligned}
& \left(1-\frac{k_{t}^{11}+k_{t}^{12}+k_{t}^{22}+k_{t}^{21}}{2}\right) 2^{-(b-1)} \sum_{h=0}^{b-1}\left(\begin{array}{c}
b-1 \\
h
\end{array}\right)\left(1-s_{t}^{1} k_{t}^{11}\right)^{h}\left(1-s_{t}^{1} k_{t}^{11}\right)^{b-1-h} 2 \alpha \theta \rho \mu \\
& +\frac{1-k_{t}^{11}-k_{t}^{12}}{2} 2^{-(b-1)} \sum_{h=0}^{b-1}\left(\begin{array}{c}
b-1 \\
h
\end{array}\right)\left(1-s_{t}^{1} k_{t}^{11}\right)^{h}\left(1-\left(1-s_{t}^{2} k_{t}^{22}\right)^{b-1-h}\right) 2(2 \alpha-1) \theta \rho \mu \\
& +\frac{k_{t}^{12}}{2} 2^{-(b-1)} \sum_{h=0}^{b-1}\left(\begin{array}{c}
b-1 \\
h
\end{array}\right)\left(1-s_{t}^{1} k_{t}^{11}\right)^{h} 2(2 \alpha-1) \theta \rho \mu-c \equiv \Lambda_{t}^{11}
\end{aligned}
$$

where $k_{t}^{11}$ and $k_{t}^{22}$ are the proportion of individuals of each type who, in the previous period, have experienced a good outcome about the good they favour; $k_{t}^{12}$ and $k_{t}^{21}$ are the proportion of individuals of each type who, in the previous period, have experienced a good outcome about the good they do not favour; $s^{1}$ and $s^{2}$ are each type's mixed signaling strategy; and $\left(\begin{array}{c}b-1 \\ h\end{array}\right)$ is the binomial coefficient ${ }_{b-1} C_{h}=(b-1) ! /((b-1-h) ! h !)$. The first term in $\Lambda_{t}^{11}$ represents the expected gain related to the possibility that, by sending the signal, the type- 1 individual induces a fully uninformed individual (of either type) to contribute towards good 1; the second term represents the expected gain related to the possibility that a signal about good 1 induces a same-type individual (of type 1) who has only received signals about good 2 to switch to good 1 from good 2; the third term represents the expected gain related to the possibility that a signal about good 2 induces a same-type individual (of type 1) who has experienced high quality in the provision of good 2 at $t-1$ to switch to good 1 from good 2 . The corresponding expected payoff for sending a truthful signal about quality of provision of good 2 would have a similar structure, but the first term would feature a factor $1-\alpha$ rather than $\alpha>1-\alpha$, and the last two terms would involve type- 2 rather than type- 1 receivers, and would feature a factor $1-2 \alpha<1$ rather than $2 \alpha-1-$ implying an expected loss. Thus, we can conclude (as initially posited) that $\Lambda_{t}^{\tau \tau^{\prime}}<\Lambda_{t}^{\tau \tau}$.

Stocks of information for type-1 individuals evolve as follows:

$$
k_{t+1}^{11}=\rho k_{t}^{11}+\rho\left(1-k_{t}^{11}\right) 2^{-b} \sum_{h=0}^{b}\left(\begin{array}{l}
b \\
h
\end{array}\right)\left(1-\left(1-s_{t}^{1} k_{t}^{11}\right)^{h}\right)
$$


$k_{t+1}^{12}=\rho k_{t}^{12} 2^{-b} \sum_{h=0}^{b}\left(\begin{array}{l}b \\ h\end{array}\right)\left(1-s^{1} k^{11}\right)^{h}+\rho\left(1-k_{t}^{11}-k_{t}^{12}\right) 2^{-b} \sum_{h=0}^{b}\left(\begin{array}{l}b \\ h\end{array}\right)\left(1-s_{t}^{1} k_{t}^{11}\right)^{h}\left(1-\left(1-s_{t}^{2} k_{t}^{22}\right)^{b-h}\right)$.

The corresponding expected payoff, $\Lambda_{t}^{22}$, for a type-2 sender, and the equalities defining the evolution of information stocks for type- 2 individuals are defined analogously.

In a stationary equilibrium, it will be the case that $\hat{k}^{11}=\hat{k}^{22}=\hat{k}^{\tau \tau}, \hat{k}^{12}=\hat{k}^{21}=\hat{k}^{\tau \tau^{\prime}}$, and $\hat{s}^{1}=\hat{s}^{2}=s$. Carrying out these substitutions, using the identities $\sum_{h=0}^{b}\left(\begin{array}{l}b \\ h\end{array}\right)=2^{b}$ and $2^{-b} \sum_{h=0}^{b}\left(\begin{array}{l}b \\ h\end{array}\right)\left(1-s k^{\tau \tau}\right)^{h}=2^{-b}\left(2-s k^{\tau \tau}\right)^{b}=\left(1-s k^{\tau \tau} / 2\right)^{b}$ - the latter derived from the identity $\sum_{h=0}^{b}\left(\begin{array}{l}b \\ h\end{array}\right) x^{h}=(1+x)^{b}-$ and further simplifying, the conditions for a stationary equilibrium with interior mixed strategies can be re-written as

$$
\begin{aligned}
& \left(\left(1-\hat{k}^{\tau \tau}-\hat{k}^{\tau \tau^{\prime}}\right)\left(1-\hat{s} \hat{k}^{\tau \tau}\right)^{b-1}+\left(1-\hat{k}^{\tau \tau}\right)\left(1-\hat{s} \hat{k}^{\tau \tau} / 2\right)^{b-1}(2 \alpha-1)\right) \Psi-c=0 \\
& \hat{k}^{\tau \tau}-\rho\left(\hat{k}^{\tau \tau}+\left(1-\hat{k}^{\tau \tau}\right)\left(1-\left(1-\hat{s} \hat{k}^{\tau \tau} / 2\right)^{b}\right)\right)=0 \\
& \hat{k}^{\tau \tau^{\prime}}-\rho\left(\hat{k}^{\tau \tau^{\prime}}\left(1-\hat{s} \hat{k}^{\tau \tau}\right)^{b}+\left(1-k^{\tau \tau}\right)\left(\left(1-\hat{s} \hat{k}^{\tau \tau} / 2\right)^{b}-\left(1-\hat{s} \hat{k}^{\tau \tau}\right)^{b}\right)\right)=0
\end{aligned}
$$

Letting $\hat{s} \hat{k}^{\tau \tau}=\hat{r}$ and solving for $\hat{k}^{\tau \tau}$ and $\hat{k}^{\tau \tau^{\prime}}$ as a function of $\hat{r}$ from (25) and (26), we obtain

$$
\begin{aligned}
& \hat{k}^{\tau \tau}=1-\frac{1-\rho}{1-\rho(1-\hat{r} / 2)^{b}}, \\
& \hat{k}^{\tau \tau^{\prime}}=1-k^{\tau \tau}-\frac{1-\rho}{1-\rho(1-\hat{r})^{b}}, \\
& \hat{k}^{\tau \tau}+\hat{k}^{\tau \tau^{\prime}}=1-\frac{1-\rho}{1-\rho(1-\hat{r})^{b}} .
\end{aligned}
$$

The total in (29) equals the expression obtained from solving for $\hat{k}$ as a function of $\hat{r}$ in a scenario with homogeneous provision.

For $\alpha=1 / 2$, both $\hat{k}^{\tau \tau}$ and $\hat{k}^{\tau \tau^{\prime}}$ come to refer to a common homogeneous stock of information; so we can write $\hat{k}^{\tau \tau}+\hat{k}^{\tau \tau^{\prime}}=\hat{k}$. Moreover, for $\alpha=1 / 2$, (24) becomes

$$
(1-\hat{k})(1-\hat{r})^{b-1} \Psi-c=0
$$

which coincides with the corresponding condition for a scenario with homogeneous pro- 
vision. So, we conclude that, for $\alpha=1 / 2$, the stationary equilibrium values of $k$ and $r$ coincide with those in a scenario with homogeneous provision, and so do those for $\alpha$ approaching $1 / 2$ from the right - the only difference being that, for $\alpha$ close to but not equal to $1 / 2$, only the stock $k^{\tau \tau}$ will give rise to information sharing, and $\hat{s}$ will equal $\hat{r} / \hat{k}^{\tau \tau}$, whereas for $\alpha=1 / 2$, all informed individuals will engage in signaling and $\hat{s}$ will equal $\hat{r} / \hat{k}$.

Substituting (27) and (29) into (30) and totally differentiating with respect to $\left(\hat{k}^{\tau \tau}+\hat{k}^{\tau \tau^{\prime}}\right)$ and $\alpha$, we obtain

$$
\frac{\mathrm{d}\left(\hat{k}^{\tau \tau}+\hat{k}^{\tau \tau^{\prime}}\right)}{\mathrm{d} \alpha}=\frac{L_{1}}{L_{2}+L_{3}}
$$

where

$$
\begin{aligned}
& L_{1}=\frac{2 b(1-\rho) \rho(1-\hat{r} / 2)^{2(b-1)}}{\left(1-\rho(1-\hat{r} / 2)^{b}\right)^{3}}>0, \\
& L_{2}=\frac{2(1-\hat{r})^{b-2}\left(b-1+\rho(1-\hat{r})^{b}\right)}{1-\rho(1-\hat{r})^{b}}>0, \\
& L_{3}=\frac{2(1-\hat{r} / 2)^{b-2}\left(b-1+\rho(1-\hat{r} / 2)^{b}\right)}{1-\rho(1-\hat{r} / 2)^{b}}>0 .
\end{aligned}
$$

Since $L_{1}, L_{2}$ and $L_{3}$ are all positive, we conclude that $\mathrm{d}\left(\hat{k}^{\tau \tau}+\hat{k}^{\tau \tau^{\prime}}\right) / \mathrm{d} \alpha$ is positive.

Thus, with reference to the implications of neighbourhood size for information sharing intensity and provision levels, this alternative specification yields qualitatively analogous predictions as the basic specification we have examined earlier - albeit with stronger signaling incentives. The main virtue of this variant, however, is that it incorporates motives that are typically thought of as being central to fundraising, namely the drive by fundraisers to steer others' contributions towards forms of provision favoured by the fundraisers themselves.

Referring back to the example presented at the end of Section 3 , for $b=2$ and $\alpha$ approaching $1 / 2$ we obtain the same level of expected provision as in the homogeneous good case, with stocks respectively equal to $\hat{k}^{\tau \tau} \simeq 0.25, \hat{k}^{\tau \tau} \simeq 0.14-$ adding up to the same information stock level, $\hat{k} \simeq 0.39$, as in the homogeneous 
good case - and $\hat{s}=0.7$ - giving the same level of information sharing intensity, $\hat{r}=\hat{s} \hat{k}^{\tau \tau} \simeq 0.18$, as in the homogeneous good case - and a level of expected provision $E\left[\hat{G}^{1}+\hat{G}^{2}\right] / N \simeq 0.195$. For $b=2$ and $\alpha=5 / 8>1 / 2$, we have $\hat{k}^{\tau \tau} \simeq 0.35$, $\hat{k}^{\tau \tau} \simeq 0.15, \hat{r} \simeq 0.29, E\left[\hat{G}^{1}+\hat{G}^{2}\right] / N \simeq 0.25>0.195$ : other things equal, heterogeneity in preferences results in a higher level of expected provision.

\section{Government subsidies}

Many developed countries offer government support to private giving - typically delivered in the form of tax relief for donations, but also involving direct support for charities and for fundraising activities.

In the equilibrium we have described private provision is the combined result of private contribution choices and private information sharing choices. Should then government policies that aim to encourage private provision be targeted towards voluntary contributions or towards fundraising (or both)?

We will look at this question in the context of a variant of our model in which private signaling costs are monetary costs that reduce private disposable income, rather than directly appearing as a non-monetary component of individuals' payoffs. This means that, if we assume that donations are bounded above to a maximum of $\mu$, private consumption by individual $i$ in period $t$ equals $1+\mu-e_{i, t}$, where $e_{i, t}$ includes all signaling costs incurred. Other than for this difference in the interpretation of signaling costs, the resulting expression for individual payoffs is exactly as before - and so is the rest of the analysis. Within this framework, we will ask what is the maximum, aggregate level of private provision that government can achieve by subsidizing contributions and then compare it with what it can achieve by subsidizing fundraising instead.

Suppose first that the government subsidizes giving. Given that the maximum contribution per individual is $\mu$, that informed individuals make contributions $\mu$, 
and that uninformed individuals make zero contributions, the best the government can do is to offer a subsidy that induces uninformed individuals to give, i.e. such that the net of subsidy price is $\theta / 2$. By doing so, it will induce all individuals (informed and uninformed) to contribute, which raises the level of expected collective provision. This, however, lowers the benefit from information sharing: the expected payoff from signaling now becomes $\left(1-k_{t}\right)\left(1-k_{t} s_{t}\right)^{b-1} \theta(\rho-1 / 2) \mu-c$, which is less than $\Lambda\left(s_{t}, k_{t}\right)$; so, as long as $c$ is not negligible, individuals will choose not to engage in information sharing: if $\left(1-k_{t}\right) \theta \rho \mu>c>\left(1-k_{t}\right) \theta(\rho-1 / 2) \mu$, then the conditions for an interior mixed-strategy equilibrium in signaling choices are met when contributions are not subsidized, whereas with subsidization of contributions the optimal signaling strategy is always $s_{t}=0$ for any $c>0$. Accordingly, information will evolve as $k_{t+1}=k_{t} \rho+\left(1-k_{t}\right) / 2$. Imposing $k_{t+1}=k_{t}$, we can then identify a stationary equilibrium information level equal to $\hat{k}^{\prime}=$ $1 /(3-2 \rho)$. Thus, government subsidies to private contributions can "crowd out" private fundraising efforts; on the other hand, by inducing uninformed individuals to give, government subsidies to contributions induce them to experiment, which results in more information being gathered through direct experimentation.

Suppose that, instead of subsidizing contributions, the government subsidizes signaling (fundraising) costs. Specifically, suppose that the government offers a subsidy $z_{t}$ that is just high enough to induce individuals to choose $s_{t}=1$, i.e. a subsidy $z_{t}$ that reduces the net-of-subsidy cost of signaling, $\left(1-z_{t}\right) c$, to a level such that $\Lambda\left(1, k_{t}\right)=0$ (once $c$ has been replaced by $\left(1-z_{t}\right) c$ ). By ensuring that $\Lambda\left(1, k_{t}\right)=0$, such a subsidy ensures that all signals are truthful. One can verify that this requires a subsidy such that $c\left(1-z_{t}\right)=\left(1-k_{t}\right)^{b}$. In this case, the stationary equilibrium condition becomes $\hat{k}=\Gamma(1, \hat{k})=\rho\left(1-(1-\hat{k})^{b+1}\right)$; this identifies a stationary equilibrium level of information $\hat{k}^{\prime \prime}$. Note that, as $b$ gets large, $\Gamma(1, \hat{k})$ converges to $\rho \hat{k}$, which gives $\hat{k}^{\prime \prime}=\rho$. In turn, for $\rho>1 / 2$, this is greater than $\hat{k}^{\prime}=1 /(3-2 \rho)$. So, for $b$ large enough, subsidizing fundraising results in a higher 
level of collective consumption than subsidizing contributions does. This result extends to finite values of $b$, as the following example shows.

Going back to the parameterization detailed at the end of Section 3, suppose now that government subsidizes contributions. The best it can do is offer a subsidy of $1 / 8$, which will result in all individuals giving. In this case, signaling effort is zero, independently of the value of $b$, and we have $\hat{k}=0.6$, and $E[\hat{G}] \simeq 0.3$. For this value of $\hat{k}$, the expected payoff from signaling is negative.

Now suppose instead that the government subsidizes signaling costs, with a subsidy just large enough to induce $\hat{s}=1$ and $\Lambda(1, \hat{k})=0$ for any given value of $\hat{k}$. Then, for $b=2$, we have $\hat{k} \simeq 0.635, E[\hat{G}] / N \simeq 0.32>0.3$. For $b=4$, we have $\hat{k} \simeq 0.664, E[\hat{G}] / N \simeq 0.331$; for $b=8$, we have $\hat{k} \simeq 0.666, E[\hat{G}] / N \simeq 0.333>0.3$.

The above conclusion does not imply that subsidies to fundraising are necessarily a more effective way of promoting private provision using a given amount of public funds. While a systematic characterization of optimal government policies under budgetary constraints is beyond the scope of the present paper, it is easy to point to scenarios where subsidizing information sharing is comparatively more effective than subsidizing giving. Suppose, for example that the government has a budget $F$ to use for this purpose, and suppose that $F<N \mu \theta / 2$, i.e. that $F$ is not large enough to fund a general (anonymous) subsidy that induces giving by uninformed individuals. In this scenario, using the available funds to subsidize giving has no effect on the volume of collective provision, whereas subsidizing signaling costs, starting from a no-subsidy equilibrium where $0<\hat{s}<0$, raises $\hat{k}$ and hence provision.

\section{Summary and discussion}

We have described a model of private provision choices in the presence of interpersonal information transmission, where private information on modes of collective 
provision can be transmitted by social proximity. Unlike in the case of private goods, informed individuals face positive incentives to engage in costly information transmission towards less informed social neighbours. In this setting information sharing has the characteristics of a local public good, even if contributions are directed towards the provision of a pure collective good.

We have shown that information sharing incentives in this model are stronger the smaller are individuals' social neighbourhoods, and this effect always dominates any advantage that larger neighbourhoods may have with respect to the diffusion of information, resulting in a lower level of provision of collective consumption at the economy level. It is worthwhile noting that, in interpreting this result in the context of real-world social connections, the size of communities need not be understood in a geographical sense. What is relevant to our arguments is the size of social communities - which include communities of co-workers, on-line communities, and the like. Thus, for example, while an increase in population density might be thought of as implying an increase in the size of geographical social neighbourhoods, it might actually lead to more rather than less information sharing if it is accompanied by a rise in the relevance of smaller, non-geographical social communities in individuals' social lives.

In addition, we have shown that, since government subsidization of contributions crowds out private information sharing effort, collective consumption is maximized by public subsidies directed to fundraising effort rather than to contributions.

We have focused on myopic information-sharing choices. To the extent that individuals are forward-looking (patient) rather than myopic, they will account for the future, non-local effects of their information efforts - which will increase the private value of information sharing but will also introduce intertemporal freeriding incentives. Nevertheless, even for agents that are forward-looking when, the size of social neighbourhoods will affect information incentives, simply be- 
cause the likelihood of any individual signal being pivotal in transmitting information in a particular time period depends on neighbourhood size.

We have also abstracted from the complications that would arise in a social networks with stable, but the mechanism we have highlighted would be present in any kind of social network. In the presence of stable links, signaling choices would also depend on individuals beliefs concerning the topological distribution of information on supplier quality across all nodes of the networks. ${ }^{17}$ Sequential equilibria with such an information structure for arbitrary network topologies are not easily tractable; nevertheless, certain basic partial-equilibrium predictions concerning the relationship between neighbourhood size and information sharing effort can still be derived that are in line with the mechanism we have formalized in our simpler setting. For example, starting from any given equilibrium featuring stationary beliefs, the unanticipated addition to the neighbourhood of $i$ of a new link to a node (a new neighbour) that $i$ 's other neighbours believe to have a nonzero probability of being informed about provision quality would unambiguously lead to less information sharing by $i$ 's pre-existing neighbours.

Our results have been derived in a framework where private contributions to collective consumption arise solely from consumption motives, but the results carry over to the case where giving stems from warm-glow motives. There are other possible interpretations of the motives for private giving that we have not examined here - such as status signaling or reciprocity effects. It should be noted, however, that these alternative interpretations of giving motives do not automatically extend to information sharing motives. Developing status signaling-based and/or reciprocity-based theories of information sharing is left for future research.

\footnotetext{
${ }^{17}$ With stable network links, signaling choices at time $t$ would depend on beliefs concerning nodes directly connected to an individual's neighbours, but the updating of beliefs about information at such nodes (on the basis of the signals observed from neighbours) would also hinge on beliefs about information at nodes further removed.
} 


\section{References}

Andreoni, J. 1990. "Impure altruism and donations to public goods: A theory of warm-glow giving," Economic Journal 100, 464-477.

Banerjee, A. 1992. "A simple model of herd behavior," Quarterly Journal of Economics 107, 797-817.

Bergstrom, T., L. Blume, and H. Varian. 1986. "On the private provision of public goods," Journal of Public Economics 29, 25-49.

Bramoullé, Y., and R. Kranton. 2007. "Public goods in networks," Journal of Economic Theory 135, 478-494.

Brown, E., and J. Ferris. 2007. "Social capital and philanthropy: An analysis of the impact of social capital on individual giving and volunteering," Nonprofit and Voluntary Sector Quarterly 36, 85-99.

Dutta, B., and K. Chatterjee. 2010. "Word of mouth advertising, credibility and learning in networks." Mimeo, University of Warwick and Pennsylvania State University.

Ellison, G. and D. Fudenberg. 1995. "Word-of-mouth communication and social learning," Quarterly Journal of Economics 110, 93-125.

Galaskiewicz, J. 1985. Social organization of an urban grants economy: A study of business philanthropy and nonprofit organizations. Orlando, Academic Press.

Gale D. and S. Kariv. 2003. "Bayesian learning in social networks," Games and Economic Behavior 45, 329-346.

Malinvaud, E. 1972. Lectures in microeconomic theory. Amsterdam, North-Holland.

UK Department for Communities and Local Government. 2010. 2008-09 Citizenship survey volunteering and charitable giving topic report. Communities and Local Government Publications, UK.

Wasserman, S., and K. Faust. 1994. Social network analysis. Methods and applications. Cambridge, Cambridge University Press. 